\title{
Prevalencia de anorexia y bulimia en adolescentes de 14 a 16 años en el Centro de Educación Media de la UAA
}

\author{
Andrade Lozano P, Barba Ruiz Esparza YA, Espejo Vásquez A, Medina de la Garza E, Moreno
} Agundis E, Terrones-Saldívar MC, Rosas Cabral A.

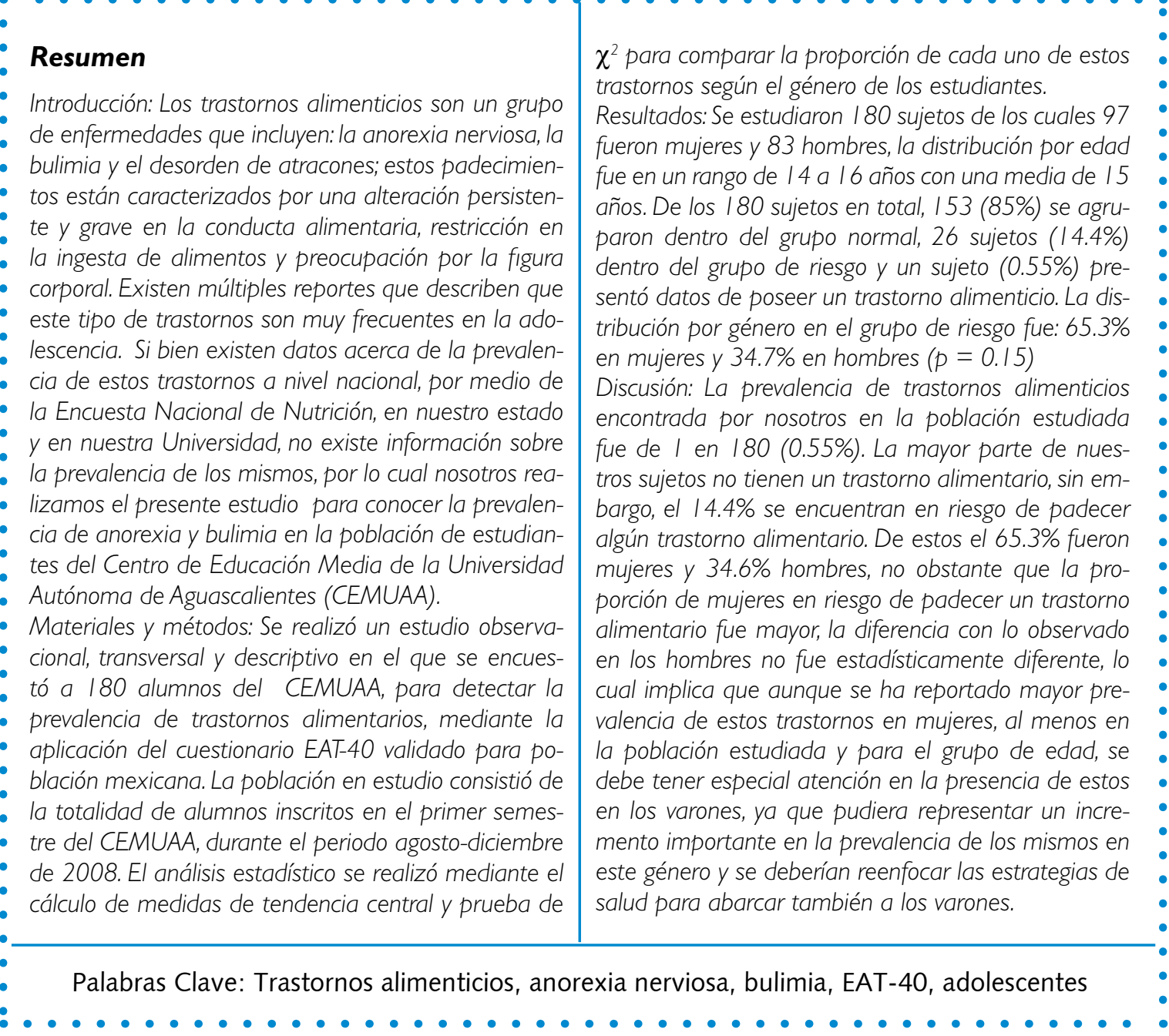

Departamento de Medicina. Centro de Ciencias Biomédicas. Universidad Autónoma de Aguascalientes. 


\section{Introducción}

Los trastornos alimenticios son un grupo de enfermedades que incluyen, la bulimia nerviosa, la anorexia nerviosa y el desorden de atracones; los cuales están caracterizados por una alteración persistente y grave de la conducta alimentaria, restricción en la ingesta de alimentos y preocupación por la figura corporal, además de sus efectos en el bienestar psicológico, estos trastornos tienen un efecto potencialmente devastador en el estado nutricio y de la salud. La anorexia nerviosa es un desorden alimenticio que usualmente inicia en la adolescencia y se caracteriza por una dieta determinada, rechazo a mantener el peso corporal en los valores mínimos normales, miedo intenso a ganar peso, realización de ejercicio de manera compulsiva, y en algunos pacientes empleo de laxantes, lo cual resulta en una rápida pérdida de peso corporal ${ }^{1,2}$.

La bulimia nerviosa es un trastorno, en el que existen episodios recurrentes de voracidad y/o atracones seguidos por conductas compensatorias inapropiadas como el vómito provocado, el abuso de diuréticos u otros medicamentos, y el ayuno o el ejercicio excesivo. Generalmente comienza durante la adolescencia, con un pico de incidencia de aparición alrededor de los 18 años, con una relación hombre a mujer de 1 a 10. La mayoría de los pacientes con bulimia tiene una condición psiquiátrica coexistente, como trastorno de ansiedad o depresión, también se ha descrito una asociación con abuso de sustancias y promiscuidad ${ }^{3}$.

Como se ha referido previamente la población adolescente es la que presenta mayor susceptibilidad a padecer este tipo de trastornos. Una de las alteraciones importantes y que causan mayor daño y secuelas a largo plazo es la presencia de amenorrea secundaria, como lo mencionan Poyastro Pinheiro y colaboradores ${ }^{4}$, de la misma manera, se ha reportado que los pacientes con anorexia nerviosa, son más propensos a sufrir depresión, trastornos del espectro autista y trastornos de ansiedad incluyendo trastornos obsesivos compulsivos. El riesgo de mortalidad en esta población es significativamente elevada y el riesgo de suicidio es pronunciado, así mismo se ha reportado que en los pacientes con bulimia, la depresión es el único factor coexistente importante pero que no tiene en esta asociación un riesgo de mortalidad aumentado ${ }^{5,6}$.

Colton y colaboradores en un estudio realizado en 2007, estudiaron adolescentes norteamericanas, del sexo femenino entre 13 y 15 años de edad y reportaron que el $14.7 \%$ de las jóvenes presentaron alteraciones alimenticias en el mes previo ${ }^{7}$; también se ha reportado que hasta el $25 \%$ de las mujeres adolescentes mexicanas tienen un riesgo significativo de padecer un desorden alimenticio y que el 6 a 7\% probablemente lo tenga ${ }^{8}$. Así mismo, se ha observado una tendencia hacia el incremento de estas alteraciones en la población de estudiantes mexicanos de secundaria y preparatoria, en especial en las mujeres, observándose un $3.4 \%$ 
de los estudiantes de secundaria con estos trastornos en 1997 y un $9.6 \%$ en $2003^{9,10}$.

En nuestro estado no existen datos publicados acerca de la prevalencia de estos trastornos. Debido a esto, y a la importancia que tiene detectar a tiempo el posible riesgo de nuestros adolescentes a padecer trastornos alimentarios, nosotros realizamos un estudio en la población perteneciente al CEMUAA para detectar la prevalencia de los mismos, mediante la aplicación del cuestionario EAT40 validado para la población mexicana 10.

| | | | | | | | | | | | | | | | | | | | | | | | | | | | | | | | | | | | | | | | | | | | | | | | | | | | | | | | | | | | | | | | | | | | | | | | | | | | | | | | | | | | | | | | | | | | | | | | | | | | | | | | | | | | |

\section{Materiales y métodos}

Se realizó un estudio transversal, descriptivo y observacional en el que se analizaron 180 alumnos del Centro de Educación Media de la Universidad Autónoma de Aguascalientes, para detectar la prevalencia de trastornos alimenticios y comparar la frecuencia de los mismos entre hombres y mujeres adolescentes.

La población estudiada consistió de la totalidad de alumnos inscritos en el primer semestre del CEMUAA, en el periodo agosto-diciembre de 2008 y que aceptaron participar en el estudio. Cada uno de los estudiantes contestó la encuesta EAT-40 validada para la población mexicana10, la cual consta de 40 ítems que se agrupan en siete factores diferentes: preocupación por la comida, imagen corporal con tendencia a la delgadez, uso y/o abuso de laxantes y presencia de vómitos, restricción alimentaria (dieta), comer lentamente, comer clandestinamente y presión social percibida para aumentar de peso.

La calificación de la escala permitió clasificar a los sujetos de estudio en tres grupos. Normal con menos de 20 puntos, grupo en riesgo de trastorno alimenticio entre $21 \mathrm{y}$ 49 puntos y grupo con trastorno alimenticio, más de 50 puntos.

El análisis estadístico se realizó mediante el cálculo de medidas de tendencia central y prueba de $\chi^{2}$ para comparar la proporción de alumnos en riesgo de padecer trastorno alimenticio según su género.

\section{| | | | | | | | | | | | | | | | | | | | | | | | | | | | | | | | | | | | | | | | | | | | | | | | | | | | | | | | | | | | | | | | | | | | | | | | | | | | | | | | | | | | | | | | | | | | | | ||}

\section{Resultados}

Se estudiaron 180 alumnos del CEMUAA, de los cuales 97 fueron mujeres y 83 hombres. El rango de edad fue entre 14 y 16 años, con promedio de 15 años, para ambos sexos.

De los 180 sujetos, 153 (85\%) calificaron dentro del grupo normal, 26 sujetos, $(14.4 \%)$ dentro del grupo de riesgo para presentar trastorno alimenticio y sólo un sujeto $(0.6 \%)$ reunió los criterios para considerarse con trastorno alimenticio.

De los 153 sujetos pertenecientes al grupo normal, $80(52.3 \%)$ fueron mujeres y 73
(47.7\%) fueron hombres, ambos con una media de edad de 15 años.

Del grupo de riesgo conformado por 26 sujetos, $17(65.3 \%)$ fueron mujeres y 9 (34.7\%) fueron hombres

Al comparar la distribución por género entre los sujetos normales y los de riesgo, encontramos una mayor proporción de mujeres en el grupo de riesgo (65.3\%) que el de hombres $(52.3 \%)$, sin embargo la diferencia no fue estadísticamente significativa, $\chi^{2}=1.53, p=0.15$. Ver tabla 1 .

De las 97 mujeres estudiadas, 80 (82.4\%) cayeron dentro del grupo normal y 17 
(17.6\%) dentro del grupo de riesgo para trastorno alimenticio.

En cuanto a los hombres, en 73 (87.9\%) fueron pertenecientes al grupo normal y 9 $(10.7 \%)$ al grupo de riesgo.

Se puede observar que una mayor propor- ción de mujeres, cae dentro del grupo con riesgo de trastorno alimenticio, en relación a los hombres (10.7\%), sin embargo al comparar estas proporciones, no existe diferencia estadísticamente significativa $\chi^{2}=$ 1.53, $p=0.15)$

\section{Tabla |}

Distribución de 180 alumnos del CEMUAA, de acuerdo al riesgo de presentar un trastorno alimentario valorados por la escala EAT40

\begin{tabular}{|c|c|c|c|}
\hline Grupo según escala EAT 40 & Femenino & Masculino & Total \\
\hline Normal & 80 & 73 & $153(85 \%)$ \\
\hline En riesgo & 17 & 9 & $26(14.4 \%)$ \\
\hline Con trastorno alimenticio & 0 & 1 & $1(0.6 \%)$ \\
\hline Total & $97(52.3 \%)$ & $83(47.7 \%)$ & $180(100 \%)$ \\
\hline
\end{tabular}

\section{Discusión}

En la literatura internacional, se reporta que para la población general, la prevalencia de trastornos alimenticios es del $14 \%{ }^{11}$. En un estudio realizado en el 2004 en Nueva York por Brewerton y cols, se reportó una prevalencia del $6 \%$ de trastornos alimenticios en una comunidad de adolescentes de preparatoria en ese estado ${ }^{11}$, sin embargo, en nuestro estudio la prevalencia es mucho menor; uno de los factores que probablemente influyó en nuestro resultado, es que los sujetos en estudio no tomaron con seriedad la resolución de la encuesta, y la información pudiera no ser totalmente verídica, a pesar de que los encuestadores explicaron de manera amplia la resolución de la misma y las posibles implicaciones que la misma tenía.

Así mismo consideramos que el instrumento empleado en este estudio, es decir, la encuesta EAT40, es fidedigna en sus resultados, ya que ha sido ampliamente utilizado en diversos estudios e incluso validada para población mexicana ${ }^{12,13}$.

Pese a lo señalado, el DSM-IV, reporta una prevalencia de estos trastornos en un rango de 0.5 a $3 \%$, señalando que las con- ductas alimenticias de riesgo son mucho más frecuentes en la población en general, haciendo referencia a todas aquellas manifestaciones similares en forma, pero menores en frecuencia e intensidad a los trastornos de conducta alimenticia ${ }^{14}$. Tal como se muestra en este estudio, la prevalencia encontrada es similar a la expuesta por el DSM-IV, sin embargo, sólo esta publicación revela esa prevalencia; y un número cada vez mayor de reportes señalan prevalecías mas elevadas especialmente en poblaciones de adolescentes ${ }^{15,16}$.

En México, la literatura denota, que se han encontrado porcentajes desde 5 hasta $18 \%$ de conductas alimenticias de riesgo en muestras de alumnos universitarios, así como adolescentes que cursan el Bachillerato en la Ciudad de México ${ }^{17}$, dato análogo al apreciado en nuestra población estudiantil, relacionado al factor de riesgo de presentar un trastorno alimenticio observado en la evaluación EAT-40, y que obligan a mantener la investigación de estos trastornos en nuestra población, para de esta manera poder detectar factores que puedan contribuir a disminuir el incremento en su presentación. 
Así mismo, al comparar la frecuencia del riesgo de presentar un trastorno alimenticio por género observamos una distribución muy similar entre hombres y mujeres, lo cual es diferente al notable predominio reportado en la literatura de estos trastornos en mujeres.

Los trastornos alimenticios típicamente se reportan en mujeres adolescentes, aunque del $5 \%$ al $15 \%$ de los casos de anorexia y bulimia nerviosa se presenta en hombres ${ }^{2}$, en el estudio que nosotros realizamos sólo encontramos un sujeto del sexo masculino con algún trastorno alimenticio $(0.55 \%)$, esto puede estar causado por la mala contestación de la encuesta y que los sujetos del sexo masculino tienen la idea de que este tipo de trastornos sólo suceden en las mujeres.

\section{Conclusiones}

La prevalencia de trastornos alimenticios que nosotros encontramos en la población estudiada fue de 1 en $180(0.55 \%)$. La mayor parte de nuestros sujetos no tienen un trastorno alimenticio, sin embargo, 26 de los $180(14.4 \%)$ sujetos se encuentra en riesgo de padecer algún trastorno alimenticio. De estos el $65.3 \%$ fueron mujeres y $34.6 \%$ hombres, no obstante la distribución no fue estadísticamente diferente $p=>0.10$.

\section{Bibliografía}

1 Yager J, Andersen AE. Anorexia nervosa. NEJM 2005; 353:1481-8.

2 Becker AE, Grinspoon SK, Libanski AK, Herzog DB. Eating disorders. NEJM 2005; 353:1481-8.

3 Mehler PS. Bulimia nervosa. NEJM 2003; 349:875-81.

4 Poyastro Pinheiro $\mathrm{P}$, Thornton LM, Plotonicov KH, Tazzi F, Klump KI, Berrettini WH, Brandt H, Crawford S. Patrones de las alteraciones menstruales en los trastornos alimenticios. Internacional Journal of Eating Disorders. 2007; 40(4).

5 Berkman ND, Lohr KN, Bulik CM, Resultados de los trastornos alimentarios: revisión sistemática de la literatura. Internacional Journal of Eating Disorders. 2007; 40(4).

6 Jáuregui-Sobera J, Santiago-Fernandez MI, EstebanezHumanes S. Eating behaviour disorders and personality. A study using MCMI-II. Aten Prim 2009:Mar 26.

7 Colton PA, Olmsted MP, Rodin GM. Alteraciones alimentarias en una población escolar de muchachas pre-adolescentes: valoración e investigación. Int J Eating Disorders. 2007; 40(5):

8 Toro J, Gómez-Pérez Mitra G, Sentís J, Valles A, Casual V, Castro J, Pineda G, León R, Platas S, Rodríguez R. Eating disorders and body image in spanish and mexican female adolescents. Soc Psichiatry Psychiatr Epidemiol. 2006; 41(7); 556-65.

9 Unikel-Santoncini C, Bojòrquez-Chapela J, Villatoro-Velásquez J, Fleiz-Bautista C, Medina-Mora Icaza ME. Risky eating behaviours in student population in Mexico City: Trends 1997-2003. Rev Inv Clin 2006; 58(1):15-27.
10 Unikel-Santoncini C, Bojòrquez-Chapela J, Carreño-Garcì $S$. Validation of a brief questionnaire to measure the risk of abnormal eating disorders. Salud Pública de Mex. 2004; 46(6): 509-15.

11 Mitchell JE, Crowl S. Medical Complications of Anorexia Nervosa and Bulimia Nervosa. Curr Opinion Psychiatry. 2006; 19: 438-443.

12 Brewerton TD. Clinical handbook of eating disorders: an integrated approach. NEJM. 2004

13 Álvarez-Rayón G, Mancilla-Díaz JM, Vázquez-Arévalo $R$, Unikel-Santoncini C, Caballero-Romo A, Mercado-Corona D. Validity of the Eating Attitudes Test: a study of Mexican eating disorders patients. PubMed. 2004 Dec; 9(4):

14 American Psychiatric Association. Diagnostic and Statistical Manual of Mental Disorders DSM-IV. 4th Edition. Washington, DC. APA. 1994

15 Olesti Baiges M, Piñol Moreso JL, Martín Vergara N, De la Fuente García M, Ricomá de Castellarnau G. Prevalencia de anorexia nerviosa, bulimia nerviosa y otros TSA en adolescentes femeninas de Reus. Anales de Pediatría. 2008; 68 (1):18-23.

16 Lenoy E. y Silber TJ. Anorexia Nerviosa en niños y adolescentes. Parte 1. Criterios diagnósticos, historia, epidemiología, etiología, fisiopatología, mortalidad y morbilidad. Arch. Argent. Pediatr. 2006; 104 (3): 253-260.

17 Unikel C, Villatoro J, Medina-Mora ME, Alcàntar EN, Fleiz C, Hernández SA. Conductas alimentarias de riesgo en adolescentes. Datos en población estudiantil del Distrito Federal. Rev Invest Clin. 2000; 52:140-147. 


\section{ANEXO 1 ENCUESTA EAT-40}

EDAD:_ SEXO: F M SEMESTRE:

Marca con una $X$ la casilla que mejor refleje su caso. Por favor contesta cuidadosamente cada pregunta. A: siempre B: casi siempre C: a menudo $D$ : a veces $E$ : pocas veces $F$ : nunca

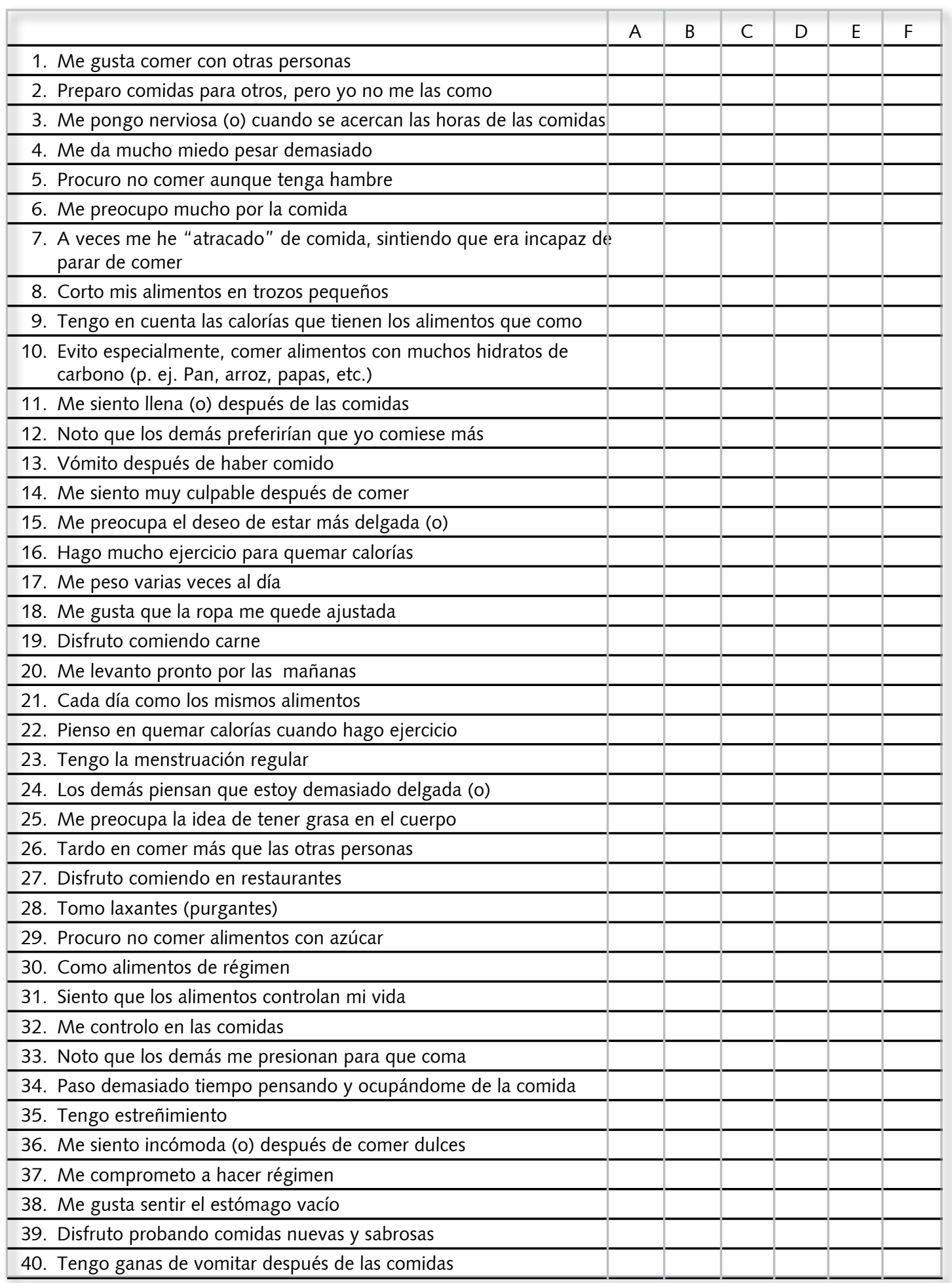

